\title{
La pharmacie hospitalière internationale : apprendre les uns des autres
}

\author{
par Rebekah J. Moles
}

A u cours des deux dernières années, le Journal canadien de la pharmacie hospitalière (JCPH) a publié la série « Perspectives internationales sur la pratique pharmaceutique » où sont présentés les systèmes de santé et la pratique de la pharmacie de divers pays des six régions de l'Organisation mondiale de la Santé (OMS) (http://www.who.int/about/regions/fr/). La série comprend des comptes rendus de l'Australie, de l'Afrique du Sud, de la Belgique, de l'Arabie saoudite, des États-Unis, du Népal, de la Jordanie, du Japon, de l'Italie, du Brésil et du Kenya (consultez l'encadré 1 dans la version anglaise de cet éditorial, à la page 3 du numéro); les derniers articles de la série, qui porteront sur la pratique à Hong Kong et en Thailande, seront publiés en 2018. Une partie de la vision du JCPH est de partager de l'information sur la "pratique de la pharmacie centrée sur le patient dans les hôpitaux et les autres milieux de soins de santé misant sur la collaboration [...] ailleurs dans le monde " : la série a ainsi été conçue pour aider à réaliser cette vision.

Les pharmaciens peuvent aussi apprendre sur la pratique à l'étranger en se rendant à des conférences internationales ou en apprenant de leurs homologues d'autres pays. La Fédération internationale pharmaceutique (FIP) s'avère un excellent point de départ. Cette organisation représente 4 millions de pharmaciens et de scientifiques du médicament. Cette organisation non gouvernementale entretient des relations officielles avec l'OMS depuis 1948. Grâce à ses partenariats et à son large réseau international en pharmacie et en sciences pharmaceutiques ainsi qu'aux innovations dans le domaine de la pratique et aux progrès scientifiques, la FIP appuie le développement de la profession de pharmacie afin de répondre aux besoins mondiaux en santél.

La structure de la FIP est complexe mais se compose essentiellement de deux organes principaux : le Conseil des sciences pharmaceutiques (Board of Pharmaceutical Sciences [BPS]) et le Conseil de la pratique pharmaceutique (Board of Pharmaceutical Practice $[\mathrm{BPP}])$. Un troisième organe prééminent, FIP Éducation (FIPEd), a été mis sur pied récemment pour veiller aux questions relatives à la formation en pharmacie. De plus, le
Young Pharmacists Group de la FIP s'adresse aux diplômés plus récents et la FIP soutient aussi l'International Pharmaceutical Students' Federation. Le BPS compte neuf groupes d'intérêts spéciaux et le BPP est composé de huit sections, dont celle de la pharmacie hospitalière. Lorsqu'une personne devient membre de la FIP, elle est affiliée à la section de son choix. Il lui est ensuite possible de s'inscrire à d'autres sections, sans restriction quant au nombre, pour la modique somme de 15 euros par section.

La section de la pharmacie hospitalière est la deuxième en importance (après celle de la pharmacie communautaire) et c'est sans doute la plus dynamique. L'objectif de la section (www.fip.org/hospital_pharmacy) est de faire évoluer chacune des dimensions de la pharmacie hospitalière, soit :

- favoriser les échanges d'idées sur des sujets professionnels qui touchent aux devoirs et responsabilités des pharmaciens d'hôpitaux;

- promouvoir les soins pharmaceutiques pour les patients dans les établissements de santé;

- favoriser les programmes de formation continue pour les pharmaciens d'hôpitaux;

- encourager l'atteinte des objectifs de la FIP lorsqu'ils s'appliquent aux pharmaciens d'hôpitaux;

- porter une attention particulière aux besoins des pays en développement;

- encourager l'intégration des services de pharmacie par la communication et la collaboration avec les autres sections.

La vision de la section pour la pharmacie hospitalière est exposée dans les déclarations de Bâle révisées sur l'avenir de la pharmacie hospitalière ${ }^{2}$ : il s'agit d'un ensemble de déclarations consensuelles conçues initialement en 2008 à la première Conférence mondiale sur l'avenir de la pharmacie hospitalière à Bâle en Suisse ${ }^{3}$, puis mises à jour en 2014 à Bangkok en Thailande ${ }^{2}$. Le travail fait pour mettre ces déclarations à jour a été décrit et leur pertinence a été résumée dans un précédent éditorial du $\mathrm{JCPH}^{4}$ et des recherches liées aux déclarations de Bâle ont aussi été publiées dans ce journal ${ }^{5,6}$. Le rôle du comité de 
recherche de la section de la pharmacie hospitalière, qui s'appelle le World Hospital Pharmacy Research Consortium, a aussi été présenté dans le $\mathrm{JCPH}^{7}$.

En plus du travail de son groupe de recherche dynamique, la section de la pharmacie hospitalière de la FIP mène aussi beaucoup d'autres activités pour atteindre ses objectifs : elle organise notamment d'excellents programmes et ateliers de formation lors des conférences annuelles de la FIP, elle offre régulièrement des webinaires et elle communique avec les membres par l'intermédiaire d'infolettres et des médias sociaux. La dernière conférence de la FIP a eu lieu à Séoul en Corée du Sud, l'édition de 2018 se tiendra à Glasgow en Écosse, puis les congressistes iront à Abu Dhabi aux Émirats arabes unis.

Le comité de direction de la section de la pharmacie hospitalière est composé d'un président, d'un président sortant, d'un trésorier, d'un secrétaire, d'un secrétaire adjoint et, pour chacune des régions de l'OMS, d'un vice-président. Tout membre de la section a la possibilité de siéger au comité de direction où les mandats sont normalement de quatre ans. Une autre façon de s'impliquer dans la section de la pharmacie hospitalière est de se joindre à l'un de ses comités : le comité de promotion des déclarations de Bâle, le comité des communications, le comité des finances, le comité d'adhésion et le comité de la recherche.

Un autre programme populaire à participation volontaire soutenu par la FIP s'appelle Pharmabridge. Il a pour objectif de renforcer les services de pharmacie dans les pays à faible revenu et les pays émergents. Il a été mis en place pour mettre en relation des personnes avec des établissements afin qu'ils échangent activement des ressources et de la formation dans les domaines de la pratique de la pharmacie, des sciences pharmaceutiques, de l'industrie pharmaceutique et de l'enseignement professionnel de la pharmacie (http://fip.org/www/index.php?page=pharmabridge). Grâce à Pharmabridge, les pharmaciens des pays en développement peuvent se rendre dans les pays développés et participer à un programme coordonné qui crée des liens organisationnels et personnels au-delà des frontières. Il s'agit d'une autre façon d'apprendre de nos collègues à l'étranger. D'ailleurs, peut-être que vous et votre établissement songerez aussi à participer à Pharmabridge dans l'avenir.

$\mathrm{Si}$, tout comme moi, vous voulez en apprendre davantage sur la pharmacie en dehors de votre milieu, alors lire abondamment, voyager à l'étranger et s'engager vous permettra vraiment de découvrir de nouvelles idées. Cela dit, j’ai remarqué une chose en lisant sur la pharmacie hospitalière exercée dans différents endroits du monde et en écoutant des conférenciers inspirants dans les congrès de la FIP : il y a toujours plus de similitudes que de différences dans notre vaste monde. En outre, nous avons tous le même but : améliorer les soins aux patients. Mais ce sont nos différences qui nous amènent à nous dépasser et à apprendre ou à essayer de nouvelles choses, ce sont elles qui nous poussent à aider les autres à atteindre leurs objectifs en leur faisant part de nos propres expériences. Si vous souhaitez en apprendre davantage à propos de la FIP et être plus orienté vers l'international, veuillez consulter le site Web de la FIP (www.fip.org) ou communiquer avec moi ou un autre membre de la section de la pharmacie hospitalière de la FIP.

[Traduction par l'éditeur]

\section{References}

1. International Pharmaceutical Federation annual report 2016: putting people first. La Haye (Pays-Bas) : International Pharmaceutical Federation; 2016. Publié au : http://fip.org/files/fip/FIP_Annual_Report_2016.pdf. Consulté le 22 décembre 2017.

2. Vermeulen LC, Moles RJ, Collins JC, Gray A, Sheikh AL, Surugue J, et al. Revision of the International Pharmaceutical Federation's Basel Statements on the future of hospital pharmacy: from Basel to Bangkok. Am J Health Syst Pharm. 2016;73(14):1077-86.

3. The Basel Statements on the future of hospital pharmacy. Am J Health Syst Pharm. 2009;66(5 Suppl 3):S61-6.

4. Moles RJ, Vermeulen L, Penm J, Ivey M. Les déclarations de Bâle : mises à jour et pertinentes pour tous [éditorial]. Can J Hosp Pharm. 2016;69(2):101-2.

5. Poh J, Vaillancourt R, Lamarre D, Oyella J. Use of the 2008 Basel consensus statements to assess, realign, and monitor pharmacy practice at a tertiary care hospital in northern Uganda: illustrative case study. Can J Hosp Pharm. 2013; 66(5):318-27.

6. Penm J, Chaar B, Moles RJ. Use of the International Pharmaceutical Federation's Basel Statements to assess and advance hospital pharmacy practice: a scoping review. Can J Hosp Pharm. 2016;69(2):131-7.

7. Moles R, Chaar B, Penm J. Le World Hospital Pharmacy Research Consortium - Suivi de la pratique dans le monde relativement aux déclarations de Bâle [éditorial]. Can J Hosp Pharm. 2014;67(5):331-2.

Rebekah J Moles, B. Pharm., Dip. Hosp. Pharm., Ph. D., Grad. Cert. Ed. Stud. (Higher Ed.), est chargé de cours principal à la Faculté de pharmacie de I'Université de Sydney, Sydney, Nouvelle-Galles du Sud, Australie. Elle est secrétaire de la section de la pharmacie hospitalière et membre des sections de l'enseignement de la pharmacie, de l'information sur la santé et les médicaments, et de la pharmacie sociale et administrative du Conseil de la pratique pharmaceutique de la Fédération internationale pharmaceutique (FIP). Elle est également rédactrice adjointe du Journal canadien de la pharmacie hospitalière.

Intérêts concurrents : Aucun déclaré

\section{Adresse de correspondance :}

$D^{\text {re }}$ Rebekah J. Moles

Faculty of Pharmacy

The University of Sydney

Pharmacy and Bank Building A15

Sydney, NSW 2006 Australia

Courriel : rebekah.moles@sydney.edu.au 